\title{
Conservative Quantization of Fast Covariance Intersection
}

\author{
Christopher Funk, Benjamin Noack, and Uwe D. Hanebeck
}

\begin{abstract}
Sensor data fusion in wireless sensor networks poses challenges with respect to both theory and implementation. Unknown cross-correlations between estimates distributed across the network need to be addressed carefully as neglecting them leads to overconfident fusion results. In addition, limited processing power and energy supply of the sensor nodes prohibit the use of complex algorithms and high-bandwidth communication. In this work, fast covariance intersection using both quantized estimates and quantized covariance matrices is considered. The proposed method is computationally efficient and significantly reduces the bandwidth required for data transmission while retaining unbiasedness and conservativeness of fast covariance intersection. The performance of the proposed method is evaluated with respect to that of fast covariance intersection, which proves its effectiveness even in the case of substantial data reduction.
\end{abstract}

\section{INTRODUCTION}

Wireless sensor networks (WSNs) comprise many autonomous but interconnected sensor nodes typically consisting of a power supply, sensors with associated data acquisition units, a processing unit, and wireless communication hardware. The sensor nodes are constrained in several respects: Available energy is severely limited, processing power is low, and wireless bandwidth has to be shared between sensor nodes [1]. In fact, wireless transmission of data is the single most energy-intensive operation performed by a sensor node, whereas the energy expenditure of processing data is comparatively low [2]. WSNs have numerous applications (cf. [3]), for instance in environmental monitoring [4], [5], [6], building automation [7], [8], or moving object tracking [9], [10].

Estimates and associated covariance matrices computed across a WSN can be combined through fusion algorithms to increase accuracy or to improve robustness to outliers. However, unknown cross-correlations between those estimates often exist. Neglecting them can cause the uncertainty of the fusion result to be underestimated. Several algorithms that mitigate the effects of unknown cross-correlations have been proposed. Examples of such algorithms are covariance intersection (CI) [11], fast covariance intersection (FCI) [12], [13], and inverse covariance intersection (ICI) [14], [15], which are guaranteed to produce results with a conservative uncertainty estimate in the form of a covariance matrix. Other algorithms such as ellipsoidal intersection (EI) [16], [17] provide no such guarantee and, on average, result in tighter approximations to the true covariance matrix.

The authors are with the Intelligent Sensor-Actuator-Systems Laboratory (ISAS), Institute of Anthropomatics and Robotics, Karlsruhe Institute of Technology (KIT), Germany. E-mail: christopher.funk@kit.edu, benjamin.noack@ieee.org, uwe.hanebeck@ieee.org
All of the above methods require the transmission of the estimates and covariance matrices to other sensor nodes and / or the fusion center to perform the fusion. Since wireless data transmission is energy-intensive, reducing the amount of transmitted data through prior compression is key to ensure long operating times of the sensor nodes in a WSN. A comprehensive survey of lossless and lossy compression methods that are suitable for WSNs is given in [18]. The surveyed methods include multiple probabilistic quantizationbased approaches [19], [20], [21], [22] tailored to estimation problems. However, only scalar measurements are considered and their respective variances are assumed to be known to the receiver. Quantization as a means of data reduction has also been applied to Kalman Filtering, a prominent example being the sign of innovations Kalman filter [23], [24]. Again, the covariance matrices associated with the measurements are assumed to be known to the receiver. In contrast to the previous works, it is assumed in [25] that the receiver has no prior knowledge of the covariance matrices, which therefore need to be transmitted via the wireless network. The authors develop data reduction methods for covariance matrices based on conservative diagonal approximations.

In this paper, similarly to [25], we assume that the covariance matrices are unknown to the receiver and therefore need to be transmitted alongside the estimates. In general, the covariance matrix will not be diagonal and dominates the amount of data that needs to be transmitted, as the number of elements grows quadratically with the dimension of the estimate. Consequently, we quantize both the covariance matrices and the estimates prior to transmission and use covariance intersection (CI) at the receiver to obtain a fused estimate. The proposed quantization scheme retains the properties of CI such as unbiasedness of the fusion result and conservativeness of the associated covariance matrix.

This paper contains three contributions: (1) We extend the probabilistic quantization method from [19], [26] to vectorvalued correlated random variables. (2) We propose a conservative covariance matrix quantization based on scalar quantization of individual matrix elements. (3) Both quantization methods are combined with fast covariance intersection to produce a conservative quantized covariance intersection (QCI) algorithm. Due to the low computational complexity of the quantization, QCI is well suited for application in resource-constrained environments.

\section{BACKGROUND}

\section{A. Notation}

Lower case letters $x \in \mathbb{R}$ denote scalar quantities and additional underlining $\underline{x} \in \mathbb{R}^{n}$ indicates $n$-dimensional 
vector-valued quantities. $n \times n$-Matrices are denoted by bold upper-case letters $\mathbf{X} \in \mathbb{R}^{n \times n}$. The $i$-th element of a vector and the $i j$-th element of a matrix are $\underline{x}^{i}$ and $\mathbf{X}^{i j}$, respectively. The use of boldface as in $\mathrm{x} \in \mathbb{R}$ and $\underline{\mathrm{x}} \in \mathbb{R}^{n}$ indicates random scalars and random vectors, respectively. Upper-case calligraphic letters $\mathcal{A}$ indicate sets. In particular, $\mathcal{S}_{+}^{n}$ is used to denote the set of symmetric positive semidefinite matrices in $\mathbb{R}^{n \times n}$. For $\mathbf{X} \in \mathcal{S}_{+}^{n}$ and $\mathbf{Y} \in \mathcal{S}_{+}^{n}$, the notation $\mathbf{X} \preceq \mathbf{Y}$ signifies that $\mathbf{Y}-\mathbf{X} \in \mathcal{S}_{+}^{n}$. If $\mathbf{X} \preceq \mathbf{Y}$ then $\mathbf{Y} \in \mathcal{S}_{+}^{n}$ is called an upper bound for $\mathbf{X} \in \mathcal{S}_{+}^{n}$. Probabilities are written as $\mathbb{P}(\cdot)$, conditional probabilities as $\mathbb{P}(\cdot \mid \cdot)$. The lower-case letter $p$ is reserved for (conditional) probability density functions, as in $p(\cdot)$ or $p(\cdot \mid \cdot)$. For (conditional) expectations the symbols $\mathbb{E}(\cdot)$ and $\mathbb{E}(\cdot \cdot \cdot)$ are used. The covariance between two random quantities is designated by $\mathbb{C}(\cdot, \cdot)$ or by $\mathbb{C}(\cdot)$ if the arguments are identical. The conditional covariance of a random quantity is denoted by $\mathbb{C}(\cdot \mid \cdot)$. For brevity, given two random vectors $\underline{\mathbf{x}} \in \mathbb{R}^{n}$ and $\mathbf{y} \in \mathbb{R}^{n}$, their covariance matrix $\mathbb{C}(\underline{\mathbf{x}}, \underline{\mathbf{y}})$ is alternatively written as $\mathbf{C}_{x y}$.

\section{B. Covariance Intersection}

The covariance intersection algorithm, originally devised by Julier and Uhlmann [11], enables the conservative fusion of two estimates of the mean of a random quantity, regardless of the generally unknown cross-covariance between the estimates. More specifically, given two unbiased estimates $\underline{\mathbf{x}}_{a} \in \mathbb{R}^{n}$ and $\underline{\mathbf{x}}_{b} \in \mathbb{R}^{n}$ of the mean $\underline{x} \in \mathbb{R}^{n}$ of some random variable and upper bounds $\hat{\mathbf{C}}_{a a}, \hat{\mathbf{C}}_{b b} \in \mathcal{S}_{+}^{n}$ of the true covariance matrices $\mathbf{C}_{a a}, \mathbf{C}_{b b} \in \mathcal{S}_{+}^{n}$ of $\underline{\mathbf{x}}_{a}$ and $\underline{\mathbf{x}}_{b}$, the covariance intersection algorithm computes an unbiased fused estimate $\underline{\mathbf{x}}_{c} \in \mathbb{R}^{n}$ and an upper bound $\hat{\mathbf{C}}_{c c} \in \mathcal{S}_{+}^{n}$ of the true covariance matrix $\mathbf{C}_{c c} \in \mathcal{S}_{+}^{n}$ of the fused estimate. Covariance intersection is conservative in the sense that the confidence ellipsoid

$$
\hat{\mathcal{E}}_{c c}=\left\{\underline{x} \in \mathbb{R}^{n} \mid\left(\underline{x}-\underline{\mathbf{x}}_{c}\right)^{\top} \hat{\mathbf{C}}_{c c}^{-1}\left(\underline{x}-\underline{\mathbf{x}}_{c}\right) \leq \epsilon\right\}
$$

induced by the upper bound $\hat{\mathbf{C}}_{c c}$ contains the confidence ellipsoid

$$
\mathcal{E}_{c c}=\left\{\underline{x} \in \mathbb{R}^{n} \mid\left(\underline{x}-\underline{\mathbf{x}}_{c}\right)^{\top} \mathbf{C}_{c c}^{-1}\left(\underline{x}-\underline{\mathbf{x}}_{c}\right) \leq \epsilon\right\}
$$

induced by $\mathbf{C}_{c c}$ for any $\epsilon>0$. For fixed $\epsilon$ this is visualized in Fig. 1.

The covariance intersection algorithm itself is defined by

$$
\begin{aligned}
\hat{\mathbf{C}}_{c c}^{-1} & =\omega \hat{\mathbf{C}}_{a a}^{-1}+(1-\omega) \hat{\mathbf{C}}_{b b}^{-1}, \\
\hat{\mathbf{C}}_{c c}^{-1} \underline{\mathbf{x}}_{c} & =\omega \hat{\mathbf{C}}_{a a}^{-1} \underline{\mathbf{x}}_{a}+(1-\omega) \hat{\mathbf{C}}_{b b}^{-1} \underline{\mathbf{x}}_{b},
\end{aligned}
$$

where any $\omega \in[0,1]$ gives an unbiased estimate and an upper bound on its true covariance matrix. The weight $\omega$ is usually determined numerically by minimizing either the trace or the determinant of $\hat{\mathbf{C}}_{c c}$. The fast covariance intersection (FCI) algorithm [12] uses the specific weight

$$
\omega=\frac{\operatorname{tr}\left(\hat{\mathbf{C}}_{b b}\right)}{\operatorname{tr}\left(\hat{\mathbf{C}}_{a a}\right)+\operatorname{tr}\left(\hat{\mathbf{C}}_{b b}\right)}
$$

that can efficiently be computed in closed form, but may result in more conservative upper bounds $\hat{\mathbf{C}}_{c c}$ compared to those obtained by using the optimal $\omega$.

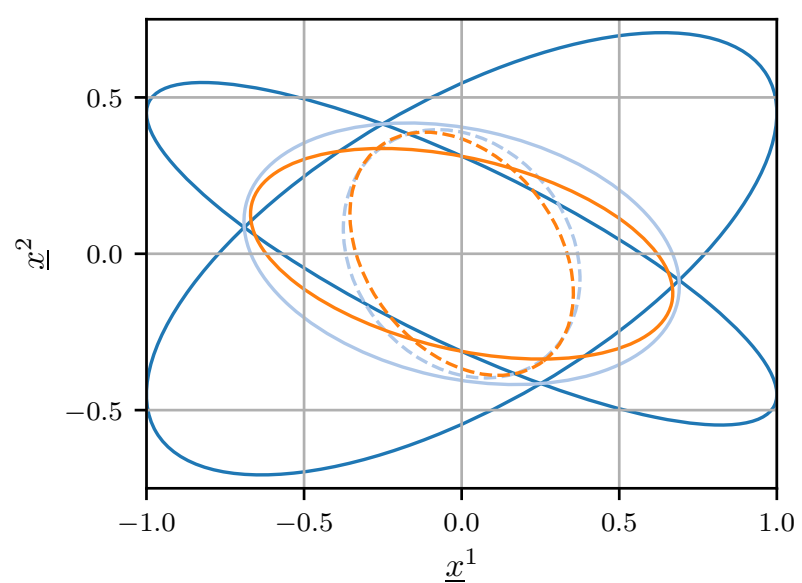

Fig. 1. Confidence ellipsoids of $\hat{\mathbf{C}}_{a a}$ and $\hat{\mathbf{C}}_{b b}$ (dark blue), $\hat{\mathbf{C}}_{c c}$ (light blue) and $\mathbf{C}_{c c}$ (dashed light blue) for exact input data. Confidence ellipsoids of $\hat{\mathbf{C}}_{c c}$ (orange) and $\mathbf{C}_{c c}$ (dashed orange) for naively quantized input data.

\section{Probabilistic Quantization}

The process of quantization maps a continuous quantity to an approximate discrete representation. For the purpose of this paper, a quantizer is a map $q: \mathcal{D} \rightarrow \mathcal{C}$, where the domain $\mathcal{D}$ is a closed, element-wise bounded subset of either $\mathbb{R}^{n}$ or $\mathbb{R}^{n \times n}$, and the codomain $\mathcal{C}$, the so-called codebook, is a finite set. If $\mathcal{D}$ is a subset of $\mathbb{R}, q$ is called a scalar quantizer. Assuming a scalar quantizer $q: \mathcal{D} \rightarrow \mathcal{C}$ with $\min (\mathcal{D}), \max (\mathcal{D}) \in \mathcal{C}$ and given a bounded random variable $\mathbf{x} \in \mathcal{D}$, the quantized random variable $q(\mathbf{x})$, in general, does not have the same mean as $\mathbf{x}$. This can be rectified by introducing randomization into the quantizer.

The randomized scalar quantizer $q_{s}: \mathcal{D}_{s} \rightarrow \mathcal{C}_{s}$ proposed in [20] (see also [26], [27]) is defined by

$$
q_{s}(\mathbf{x})=\left\{\begin{array}{ll}
\lfloor\mathbf{x}\rfloor & \text { with probability } \frac{\lceil\mathbf{x}\rceil-\mathbf{x}}{\delta_{s}} \\
\lceil\mathbf{x}\rceil & \text { with probability } 1-\frac{\lceil\mathbf{x}\rceil-\mathbf{x}}{\delta_{s}}
\end{array} .\right.
$$

The brackets $\lceil\cdot\rceil$ and $\lfloor\cdot\rfloor$ indicate rounding up to and rounding down to the next codeword (element of the codebook), respectively. The codebook is given by

$$
\mathcal{C}_{s}=\left\{x_{\max }-k \delta_{s} \mid 0 \leq k<2^{b}\right\},
$$

where $x_{\max }$ is the maximum codeword, $\delta_{s}=x_{\max } / 2^{b-1}$ is the increment between adjacent codewords, and $b$ is the number of bits required to represent a codeword. Note that the assumption $\min \left(\mathcal{D}_{s}\right), \max \left(\mathcal{D}_{s}\right) \in \mathcal{C}_{s}$ is crucial for $q_{s}$ to be well defined. The quantizer in (1) satisfies [20]

$$
\begin{aligned}
& \mathbb{E}\left(q_{s}(\mathbf{x})\right)=\mathbb{E}(\mathbf{x}) \\
& \mathbb{C}\left(q_{s}(\mathbf{x})\right) \leq \mathbb{C}(\mathbf{x})+\delta_{s}^{2}
\end{aligned}
$$

that is, it provides an unbiased estimate of the scalar input and there is an upper bound on the variance of the estimate.

\section{Considered PRoblem}

We apply covariance intersection to quantized estimates and covariance matrix bounds in order to reduce the amount of transmitted data in a WSN. This reduces the power consumption and extends the operating life of the sensor nodes. 
However, applying covariance intersection to quantized data is not straightforward. Deterministic quantization of the estimates introduces bias and additional noise, which causes (1) biased fusion results and (2) invalid covariance matrix bounds. In addition, naive element-wise quantization of the covariance matrix bounds can lead to quantized matrices that are no longer valid upper bounds, or for that matter, not even valid covariance matrices in the sense of being symmetric positive semidefinite. Fig. 1 illustrates the repercussions of naive quantization for the results of covariance intersection. Clearly, the upper bound computed by applying covariance intersection to naively quantized data is not conservative.

The issue of bias can be addressed by using probabilistic quantization for the estimates. Probabilistic quantization has been introduced in Sec. II-C for the scalar case and will be extended to the vector-valued case in Sec. IV-A. The issue of conservative quantization of covariance matrix bounds can be approached by accounting for the noise introduced by the quantization of the estimates and by enforcing certain conditions on the quantization errors of the covariance matrix bounds. This will be discussed in detail in Sec. IV-B.

\section{QuANTIZED COVARIANCE INTERSECTION}

\section{A. Unbiased Estimate Quantization}

In this section, the unbiasedness and variance bound results for the probabilistic quantization approach reviewed in Sec. II-C are extended to element-wise bounded random vectors with potentially correlated entries. It is shown that element-wise application of the aforementioned approach results in unbiasedness of the quantized vectors. An upper bound for the covariance matrix of the quantized random vector, analogous to the scalar bound in (3), is derived.

The proposed probabilistic quantizer $q_{m}: \mathcal{D}_{m} \rightarrow \mathcal{C}_{m}$ for element-wise bounded random vectors $\underline{\mathrm{x}} \in \mathcal{D}_{m} \subset$ $\mathbb{R}^{n}$ is an element-wise version of the one given by (1). Correspondingly, its domain and codebook are the Cartesian products $\mathcal{D}_{m}=\mathcal{D}_{s}^{n}$ and $\mathcal{C}_{m}=\mathcal{C}_{s}^{n}$ of the scalar domain $\mathcal{D}_{s}$ and codebook $\mathcal{C}_{s}$ from Sec. II-C. Each element of a random input vector $\underline{\mathbf{x}}$ is quantized according to (1), resulting in the random output vector $\mathbf{y}=q(\underline{\mathbf{x}})$. This quantization process can be described by the conditional probabilities

$$
\begin{aligned}
\mathbb{P}(\underline{\mathbf{y}} \mid \underline{\mathbf{x}})=\prod_{i=1}^{n} \mathbb{P}\left(\underline{\mathbf{y}}^{i} \mid \mathbf{x}\right), \\
\mathbb{P}\left(\underline{\mathbf{y}}^{i} \mid \underline{\mathbf{x}}\right)=\left\{\begin{array}{ll}
\frac{\left\lceil\underline{\mathbf{x}}^{i}\right\rceil-\underline{\mathbf{x}}^{i}}{\delta_{s}}, & \underline{\mathbf{y}}^{i}=\left\lfloor\underline{\mathbf{x}}^{i}\right\rfloor \\
1-\frac{\left\lceil\underline{\mathbf{x}}^{i}\right\rceil-\underline{\mathbf{x}}^{i}}{\delta_{s}}, & \underline{\mathbf{y}}^{i}=\left\lceil\underline{\mathbf{x}}^{i}\right\rceil
\end{array} .\right.
\end{aligned}
$$

As before, $\lceil\cdot\rceil$ rounds up to and $\lfloor\cdot\rfloor$ rounds down to the next codeword in the scalar codebook $\mathcal{C}_{s}$ and $\delta_{s}$ is the distance between adjacent codewords in said codebook.

The next corollary is an immediate consequence of (2) applied element-wise to the random vector $\mathbf{y}=q(\underline{\mathbf{x}})$.

Corollary 1 (Unbiasedness): Let $q_{m}: \mathcal{D}_{m}^{-} \rightarrow \mathcal{C}_{m}$ be the quantizer defined by (4) and (5). Let $\underline{\mathrm{x}} \in \mathcal{D}_{m}$ be a random vector. Then $\mathbb{E}\left(q_{m}(\underline{\mathbf{x}})\right)=\mathbb{E}(\underline{\mathbf{x}})$ holds.
Due to quantization, the random vector $\underline{\mathbf{y}}=q_{m}(\underline{\mathbf{x}})$ contains additional noise compared to the input $\underline{x}$. Consequently, the covariance matrix of the input must be adapted to reflect the increased uncertainty due to quantization. In general, computing the exact covariance matrix of $\mathbf{y}$ is infeasible without knowledge of the distribution ${ }^{1}$ of $\underline{\underline{x}}$. Therefore, a conservative upper bound for $\mathbb{C}(\underline{\mathbf{y}})$ in the same vein as (3), is determined.

Theorem 1 (Upper Bound): Let $q_{m}: \mathcal{D}_{m} \rightarrow \mathcal{C}_{m}$ be the quantizer defined by (4) and (5). Let $\underline{\mathrm{x}} \in \mathcal{D}_{m}$ be a random vector. Then $\mathbb{C}\left(q_{m}(\underline{\mathbf{x}})\right) \preceq \mathbb{C}(\underline{\mathbf{x}})+\delta_{s}^{2} \mathbf{I}$ holds.

Proof: As can easily be checked using (5) and $\left\lceil\underline{\mathrm{x}}^{i}\right\rceil-\delta_{s}=$ $\left\lfloor\underline{\mathbf{x}}^{i}\right\rfloor$, the conditional mean of $\underline{\mathbf{y}}^{i}$ is given by $\mathbb{E}\left(\underline{\mathbf{y}}^{i} \mid \underline{\mathbf{x}}\right)=\underline{\mathbf{x}}^{i}$ from which $\mathbb{E}(\mathbf{y} \mid \underline{\mathbf{x}})=\underline{\mathbf{x}}$ follows. The conditional covariance of $\underline{\mathbf{y}}$ given $\underline{\mathbf{x}}$ then has zero off-diagonal $(i \neq j)$ elements

$$
\begin{aligned}
\mathbb{C}(\underline{\mathbf{y}} \mid \underline{\mathbf{x}})^{i j} & =\mathbb{E}\left(\left(\underline{\mathbf{y}}^{i}-\underline{\mathbf{x}}^{i}\right)\left(\underline{\mathbf{y}}^{j}-\underline{\mathbf{x}}^{j}\right) \mid \underline{\mathbf{x}}\right) \\
& =\mathbb{E}\left(\underline{\mathbf{y}}^{i}-\underline{\mathbf{x}}^{i} \mid \mathbf{x}\right) \mathbb{E}\left(\underline{\mathbf{y}}^{j}-\underline{\mathbf{x}}^{j} \mid \mathbf{x}\right)=0
\end{aligned}
$$

due to conditional independence. Straightforward computation using (5) and $\left\lceil\underline{\mathbf{x}}^{i}\right\rceil-\delta_{s}=\left\lfloor\underline{\mathbf{x}}^{i}\right\rfloor$ shows that the diagonal elements of $\mathbb{C}(\underline{\mathbf{y}} \mid \underline{\mathbf{x}})$ are given by

$$
\mathbb{C}(\underline{\mathbf{y}} \mid \underline{\mathbf{x}})^{i i}=\mathbb{E}\left(\left(\underline{\mathbf{y}}^{i}-\underline{\mathbf{x}}^{i}\right)^{2} \mid \underline{\mathbf{x}}\right)=\delta_{s} \underline{\mathbf{e}}^{i}-\left(\underline{\mathbf{e}}^{i}\right)^{2},
$$

where $\underline{\mathbf{e}}^{i}=\left\lceil\underline{\mathbf{x}}^{i}\right\rceil-\underline{\mathbf{x}}^{i}$. The law of total covariance states that $\mathbb{C}(\underline{\mathbf{y}})=\mathbb{C}(\mathbb{E}(\underline{\mathbf{y}} \mid \underline{\mathbf{x}}))+\mathbb{E}(\mathbb{C}(\underline{\mathbf{y}} \mid \underline{\mathbf{x}}))$, which results in

$$
\mathbb{C}(\underline{\mathbf{y}})=\mathbb{C}(\underline{\mathbf{x}})+\mathbb{E}(\mathbb{C}(\underline{\mathbf{y}} \mid \underline{\mathbf{x}}))
$$

due to conditional unbiasedness of $\mathbf{y}$ given $\underline{x}$. Note that $\underline{\mathbf{e}}^{i}<\delta_{s}$ and $0 \leq\left(\underline{\mathbf{e}}^{i}\right)^{2}$ hold. This gives upper bounds on the expectation of the diagonal entries of the conditional covariance matrix: $\delta_{s} \mathbb{E}\left(\underline{\mathbf{e}}^{i}\right)-\mathbb{E}\left(\left(\underline{\mathbf{e}}^{i}\right)^{2}\right) \leq \delta_{s}^{2}$. Due to these bounds and because $\mathbb{E}(\mathbb{C}(\underline{\mathbf{y}} \mid \underline{\mathbf{x}}))$ is diagonal, the upper bound

$$
\mathbb{C}(\underline{\mathbf{y}}) \preceq \mathbb{C}(\underline{\mathbf{x}})+\delta_{s}^{2} \mathbf{I}
$$

holds for the covariance of $\underline{\mathbf{y}}$.

The upper bound given above is fast to compute and does not require any knowledge of the distribution of the input vector $\underline{x}$. However, it overestimates the true covariance matrix of the quantized vector especially when a component of $\underline{\mathrm{x}}$ is concentrated between two codewords or for large $\delta_{s}$.

\section{B. Conservative Covariance Quantization}

In the following, a conservative quantizer for covariance matrices, i.e., symmetric positive semidefinite matrices, is derived. To that end, let $q_{c}: \mathcal{D}_{c} \rightarrow \mathcal{C}_{c}$ be a quantizer that maps symmetric positive semidefinite matrices from an element-wise bounded and closed domain $\mathcal{D}_{c} \subset \mathcal{S}_{+}^{n}$ to a finite codebook $\mathcal{C}_{c} \subset \mathbb{R}^{n \times n}$. The quantizer map $q_{c}: \mathcal{D}_{c} \rightarrow$ $\mathcal{C}_{c}$ should satisfy the conservativeness condition

$$
\forall \mathbf{X} \in \mathcal{D}_{c}: \mathbf{X} \preceq q_{c}(\mathbf{X})
$$

to ensure that the quantized matrix $q_{c}(\mathbf{X})$ is an upper bound of the original matrix $\mathbf{X}$. With the quantization error

\footnotetext{
${ }^{1}$ If the distribution of $\underline{\mathbf{x}}$ was known, the approach in [28], which is based on a series expansion, could be used to approximate $\mathbb{C}(\underline{\mathbf{y}})$ arbitrarily well.
} 
matrix defined as $\boldsymbol{\Delta}=q_{c}(\mathbf{X})-\mathbf{X}$, the following lemma gives an alternative characterization of the requirement for conservativeness expressed by (6).

Lemma 1: Let $q_{c}:: \mathcal{D}_{c} \rightarrow \mathcal{C}_{c}$ and $\boldsymbol{\Delta}$ be as above, then it holds that $\forall \mathbf{X} \in \mathcal{D}_{c}: \mathbf{X} \preceq q_{c}(\mathbf{X}) \Longleftrightarrow \mathbf{0} \preceq \Delta(\mathbf{X})$.

Proof: From (6) and $\boldsymbol{\Delta}=q_{c}(\mathbf{X})-\mathbf{X}$ it follows that $\mathbf{X} \preceq$ $\mathbf{X}+\boldsymbol{\Delta}$. Then $\mathbf{0} \preceq \boldsymbol{\Delta}$ holds by definition of $\preceq$. Assuming $\mathbf{0} \preceq \boldsymbol{\Delta}$ and $\mathbf{X} \in \overline{\mathcal{S}}_{+}^{n}, \mathbf{X} \preceq \mathbf{X}+\boldsymbol{\Delta}$ follows immediately.

In other words, the quantization error matrix must be symmetric positive semidefinite for the quantized matrix to be an upper bound of the original matrix. A simple sufficient condition for that is the quantization error matrix being diagonally dominant, as described in the next theorem, which follows directly from the Gershgorin circle theorem [29].

Theorem 2 (Diagonal Dominance): Let $\boldsymbol{\Delta} \in \mathbb{R}^{n \times n}$ be symmetric and diagonally dominant, i.e., let it satisfy

$$
\left|\Delta^{i i}\right| \geq \sum_{j=1 \neq i}^{n}\left|\Delta^{i j}\right|
$$

for $i=1 \ldots n$, then $\mathbf{0} \preceq \Delta$ holds.

The approach to conservative quantization of a symmetric positive semidefinite matrix $\mathbf{X} \in \mathcal{D}_{c}$ pursued here is to first quantize the off-diagonal elements of $\mathbf{X}$ using a codebook $\mathcal{C}_{O}$ and to then quantize the diagonal elements using a codebook $\mathcal{C}_{d}$ such that Ineqs. (7) are satisfied. This leads to a quantizer

$$
q_{c}(\mathbf{X})^{i j}=\left\{\begin{array}{ll}
\left\lceil\mathbf{X}^{i j}+\sum_{k=1 \neq i}^{n}\left|\operatorname{rd}\left(\mathbf{X}^{i k}\right)-\mathbf{X}^{i k}\right|\right], & i=j \\
\operatorname{rd}\left(\mathbf{X}^{i j}\right), & i \neq j
\end{array},\right.
$$

where $\operatorname{rd}(\cdot)$ rounds to the nearest codeword in the offdiagonal codebook $\mathcal{C}_{O}$ and $\lceil\cdot\rceil$ rounds up to the nearest codeword in the diagonal codebook $\mathcal{C}_{d}$. The codebooks are given by

$$
\begin{aligned}
& \mathcal{C}_{o}=\left\{x_{\text {max }}-k \delta_{o} \mid 0 \leq k<2^{b}\right\}, \\
& \mathcal{C}_{d}=\left\{x_{\text {max }}+(n-1) \delta_{o} / 2-k \delta_{d} \mid 0 \leq k<2^{b}\right\}
\end{aligned}
$$

where $x_{\max }$ is the maximum off-diagonal codeword, $\delta_{o}=$ $x_{\max } / 2^{b-1}$, and $\delta_{d}=\left(x_{\max }+(n-1) \delta_{o} / 2\right) /\left(2^{b}-1\right)$ with $b$ the number of bits per codeword. The quantizer $q_{c}$ is well defined as long as the off-diagonal elements of the matrices in $\mathcal{D}_{c}$ are in the interval $\left[\min \left(\mathcal{C}_{o}\right), \max \left(\mathcal{C}_{o}\right)\right]$ and the diagonal elements are smaller than or equal to $\max \left(\mathcal{C}_{o}\right)$. This is because $\mathcal{C}_{d}$ is defined such that, even for the worst possible off-diagonal quantization errors, there always are sufficiently large codewords to round up to when quantizing the diagonal elements. When not stated otherwise, the above conditions for well-defined $q_{c}$ are implicitly assumed to hold. The next theorem confirms that the output of $q_{c}$ is indeed an upper bound for its input.

Theorem 3 (Conservativeness): The quantizer $q_{c}: \mathcal{D}_{c} \rightarrow$ $\mathcal{C}_{c}$ as in (8) has positive semidefinite quantization error matrix $\boldsymbol{\Delta}=q_{c}(\mathbf{X})-\mathbf{X}$.
Proof: The off-diagonal quantization errors are $\boldsymbol{\Delta}^{i j}=$ $\operatorname{rd}\left(\mathbf{X}^{i k}\right)-\mathbf{X}^{i k}$ and the diagonal quantization errors are

$$
\boldsymbol{\Delta}^{i i}=\left\lceil\mathbf{X}^{i i}+\sum_{k=1 \neq i}^{n}\left|\boldsymbol{\Delta}^{i k}\right|\right\rceil-\mathbf{X}^{i i}
$$

By the definition of $\lceil\cdot\rceil$ we have

$$
\boldsymbol{\Delta}^{i i} \geq \sum_{k=1 \neq i}^{n}\left|\boldsymbol{\Delta}^{i k}\right|
$$

and the claim follows from Theorem 2 .

Furthermore, the quantizer defined by (8) is optimal in the sense that, given the codebooks $\mathcal{C}_{d / o}$, there is no quantizer with symmetric diagonally dominant quantization error matrix $\boldsymbol{\Delta}$ that has smaller Frobenius norm $\|\cdot\|_{F}$ of $\boldsymbol{\Delta}$.

Theorem 4 (Optimality): Let $q_{c}: \mathcal{D}_{c} \rightarrow \mathcal{C}_{c}$ be defined by (8) with element-wise codebooks $\mathcal{C}_{d}$ and $\mathcal{C}_{o}$ defined by (10) and (9). Given $\mathbf{X} \in \mathcal{D}_{c}$, the quantization error matrix $\boldsymbol{\Delta}=$ $q_{c}(\mathbf{X})-\mathbf{X}$ is the optimal solution of

$$
\begin{array}{cll}
\underset{\Delta \in \mathbb{R}^{n \times n}}{\operatorname{minimize}} & \|\boldsymbol{\Delta}\|_{F}^{2} \\
\text { subject to } & \sum_{j=1 \neq i}^{n}\left|\boldsymbol{\Delta}^{i j}\right| \leq \boldsymbol{\Delta}^{i i} & \forall i=1 \ldots n \\
& \mathbf{X}^{i i}+\boldsymbol{\Delta}^{i i} \in \mathcal{C}_{d} & \forall i=1 \ldots n \\
& \mathbf{X}^{i j}+\boldsymbol{\Delta}^{i j} \in \mathcal{C}_{o} & \forall i \neq j
\end{array}
$$

Proof: The problem can be reformulated as a nested minimization, the inner one being over the diagonal elements and the outer one being over the off-diagonal elements. The inner minimization can be split into decoupled minimizations

$$
\begin{array}{cl}
\underset{\boldsymbol{\Delta}^{i i} \in \mathbb{R}}{\operatorname{minimize}} & \left|\boldsymbol{\Delta}^{i i}\right|^{2} \\
\text { subject to } & \sum_{j=1 \neq i}^{n}\left|\boldsymbol{\Delta}^{i j}\right| \leq \boldsymbol{\Delta}^{i i} \\
& \mathbf{X}^{i i}+\boldsymbol{\Delta}^{i i} \in \mathcal{C}_{d}
\end{array}
$$

for $i=1, \ldots, n$. By definition of the $\lceil\cdot\rceil$ operation $\boldsymbol{\Delta}^{i i *}=$ $\left[\mathbf{X}^{i i}+\sum_{j=1 \neq i}^{n}\left|\boldsymbol{\Delta}^{i j}\right|\right]-\mathbf{X}^{i i}$ are the optimal solutions to these subproblems. They exist because $q_{c}$ is well defined. The minimum cost of each decoupled problem is thus

$$
\left.\left|\boldsymbol{\Delta}^{i i *}\right|^{2}=|| \mathbf{X}^{i i}+\sum_{j=1 \neq i}^{n}\left|\boldsymbol{\Delta}^{i j}\right|\right\rceil-\left.\mathbf{X}^{i i}\right|^{2}
$$

which is non-decreasing in each $\left|\Delta^{i j}\right|$. Using this intermediate result, the outer minimization problem is given by

$$
\begin{array}{lll}
\underset{\boldsymbol{\Delta}^{i j} \in \mathbb{R}, i \neq j}{\operatorname{minimize}} & \sum_{i=1}^{n}\left|\boldsymbol{\Delta}^{i i *}\right|^{2}+\sum_{i=1}^{n} \sum_{j=1 \neq i}^{n}\left|\boldsymbol{\Delta}^{i j}\right|^{2} & \\
\text { subject to } & \mathbf{X}^{i j}+\boldsymbol{\Delta}^{i j} \in \mathcal{C}_{o} & \forall i \neq j
\end{array}
$$

and its minimum is obtained by minimizing the $\left|\boldsymbol{\Delta}^{i j}\right|^{2}$ separately, as due to the non-decreasing property, $\left|\boldsymbol{\Delta}^{i i *}\right|^{2}$ is minimal if each $\left|\boldsymbol{\Delta}^{i j}\right|^{2}$ is minimal. Thus the minimum is, 
by definition of $\operatorname{rd}(\cdot)$, attained by setting $\boldsymbol{\Delta}^{i j *}=\operatorname{rd}\left(\mathbf{X}^{i j}\right)$. Although the above approach minimizes the conservativeness of the quantized matrix in the sense of Theorem 4, the inequalities in (7) are only a sufficient condition for the quantization error matrix to be positive semidefinite. Hence, the results of this method are usually more conservative than strictly necessary. Note that the proposed quantization method can be computed efficiently thanks to the individual quantization of the matrix elements.

\section{Application to Covariance Intersection}

By quantizing the estimates and upper bounds used as input data for fast covariance intersection with the methods from Sec. IV-A and Sec. IV-B unbiasedness and conservativeness is retained. Consequently, the output of FCI when applied to the quantized data is unbiased and conservative as well. The entire process can be summarized as follows:

1) Quantize estimates $\underline{\mathbf{x}}_{a}$ and $\underline{\mathbf{x}}_{b}$ and adapt their upper bounds $\hat{\mathbf{C}}_{a a}$ and $\hat{\mathbf{C}}_{b b}$ using the approach in Sec. IV-A.

2) Quantize the adapted upper bounds from the previous step using the approach from Sec. IV-B.

3) Transmit the quantized data.

4) Apply fast covariance intersection to the quantized estimates and quantized adapted upper bounds.

It should be noted that covariance intersection could be used instead of fast covariance intersection, at the expense of increased computational cost. In the following, we use the term quantized covariance intersection (QCI) to refer to the combination of fast covariance intersection with the introduced quantization schemes.

\section{Results AND Discussion}

The quantization error of the proposed covariance matrix quantizer is evaluated using randomly selected covariance matrices. In addition, the performance of QCI relative to FCI is evaluated a) by applying both methods to randomly generated data and b) in a $2 \mathrm{D}$ target tracking scenario. Evaluation a) demonstrates the behaviour of QCI with respect to coarseness of quantization and dimensionality of the input data. Evaluation b) benchmarks QCI's particular performance for a given target trajectory and varying coarseness of quantization.

\section{A. Evaluation of Covariance Quantizer}

The covariance matrix quantizer from Sec. IV-B is applied to independent samples of random covariance (symmetric positive semidefinite) matrices. The random matrices are generated as $\mathbf{X}=\mathbf{L L}^{\top}$ where $\mathbf{L} \in \mathbb{R}^{n \times n}$ has zeromean, normally distributed elements with variance one. It should be noted that $\mathbf{X}$ is not uniformly distributed. The Frobenius norms of the resulting quantization error matrices are averaged over all samples.

Fig. 2 shows the aforementioned averaged Frobenius norms for varying numbers of bits per codeword $b$ and several matrix dimensions $n$. The codebook parameter $x_{\max }=50.0$ was used and 10000 samples were included in the average.

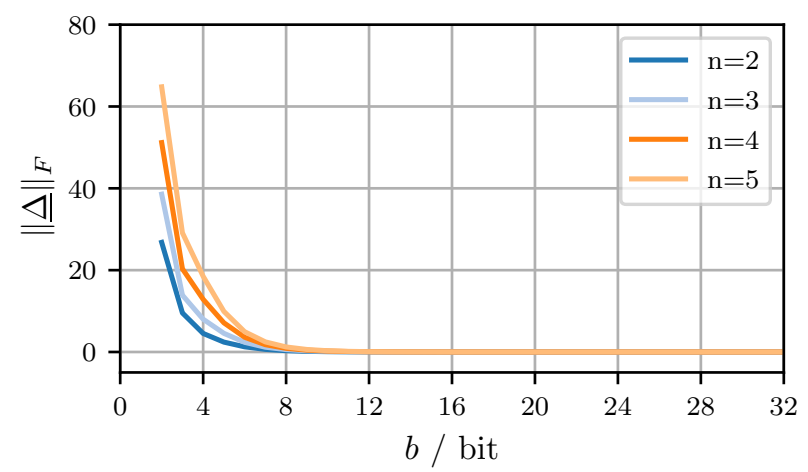

Fig. 2. Frobenius norm $\|\Delta\|_{F}$ of quantization error matrix $\Delta$ for varying dimensions $n$ and bits per scalar $b$.

The quantization error is negligible for $b$ greater than eight bits per codeword. Below that threshold the error increases, with larger $n$ being affected more strongly. The general trend with respect to $b$ is a result of the quantization steps $\delta_{o / d}$ increasing exponentially for decreasing $b$. The dependency on $n$ is caused by two factors. On the one hand the quantization error per element remains roughly the same, whereas the number of elements increases quadratically. On the other hand the accumulated off-diagonal quantization error that is added to the diagonal elements to ensure conservativeness, grows larger with increasing $n$. This contributes to a larger diagonal quantization error and thus a larger error overall.

\section{B. Evaluation of QCI - Randomly Generated Data}

Input data for QCI / FCI is generated by first drawing an element-wise Gaussian random matrix $\mathbf{L} \in \mathbb{R}^{2 n \times 2 n}$ whose elements have mean zero and variance one. Then a random vector $\underline{\mathbf{x}}=\left[\underline{\mathbf{x}}_{a}^{\top}, \underline{\mathbf{x}}_{b}^{\top}\right]^{\top} \in \mathbb{R}^{2 n}$, where $\underline{\mathbf{x}}_{a} \in \mathbb{R}^{n}$ and $\underline{\mathbf{x}}_{b} \in \mathbb{R}^{n}$, is drawn from a normal distribution with mean zero and conditional covariance matrix $\mathbb{C}(\underline{\mathbf{x}} \mid \mathbf{L})=\mathbf{L} \mathbf{L}^{\top}$.

FCI and QCI are applied to the thus-generated vectors $\underline{\mathbf{x}}_{a}$ and $\underline{\mathbf{x}}_{b}$ using $\mathbb{C}\left(\underline{\mathbf{x}}_{a} \mid \mathbf{L}\right)$ and $\mathbb{C}\left(\underline{\mathbf{x}}_{b} \mid \mathbf{L}\right)$ as (obviously exact) upper bounds for their conditional covariance matrices. The actual mean squared error (MSE) of the estimates produced by QCI and FCI is computed by repeatedly generating new input data and averaging the squared Euclidean norm of the resulting estimates. The traces of the resulting covariance upper bounds, which are upper bounds for the actual MSEs, are also calculated for each random input and averaged. The computed actual MSE and the averaged trace are discarded if the quantization of any of the involved covariance matrices fails due to the limited range of the codebooks.

Fig. 3 shows the relative increase of the actual MSE and of the averaged trace, when using QCI instead of FCI. Varying dimensions $n$ and numbers of bits per codeword $b$ are considered. The results were obtained by averaging over 10000 independent trials and by setting the parameter $x_{\max }$ of the scalar codebooks $\mathcal{C}_{m}, \mathcal{C}_{d}$, and $\mathcal{C}_{o}$ to $x_{\max }=50.0$.

Fig. 3 indicates that the increase in actual MSE and in the average trace of the covariance upper bounds is insignificant except for small $b$. The dimensionality of the input data has little influence on performance, although the performance slightly improves for larger $n$. The increase in the averaged 


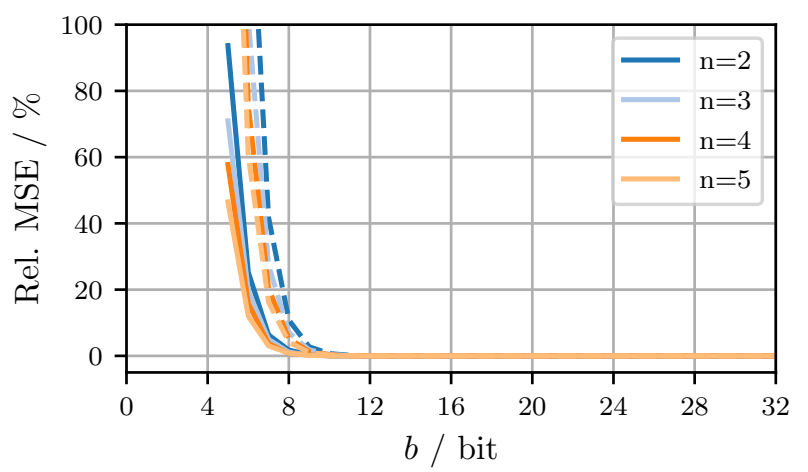

Fig. 3. Relative increase of actual MSE (solid) / averaged trace (dashed) of QCI with respect to FCI for varying dimensions $n$ and bits per scalar $b$.

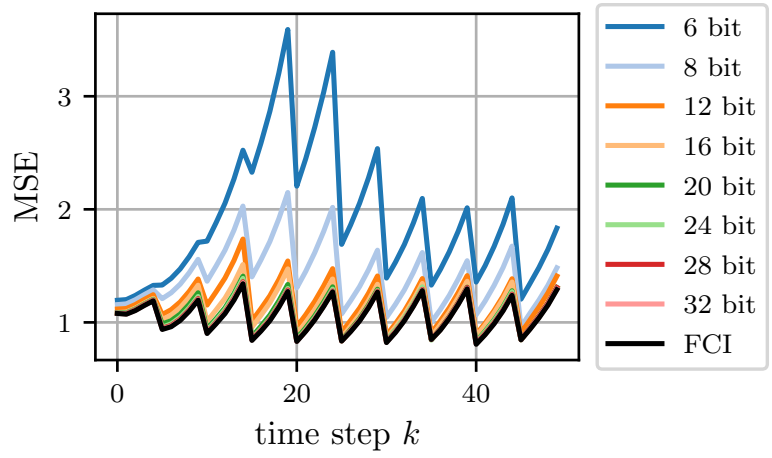

Fig. 4. The mean-squared estimation error of sensor node $b$ for varying bits per scalar.

traces of the covariance upper bounds is larger than the increase in actual MSE for all $n$ and $b$. This is to be expected, since the quantization process retains conservativeness. The quantization of the covariance matrices fails below five bits per scalar, due to excessive inflation of the covariance upper bounds of the inputs by the probabilistic quantization.

\section{Evaluation of $Q C I-2 D$ Tracking Scenario}

This evaluation scenario considers two sensor nodes that cooperatively track an object. The object is characterized by a discrete-time (nearly) constant acceleration model

$$
\underline{\mathbf{x}}_{k+1}=\left[\begin{array}{cc}
\mathbf{A} & \mathbf{0} \\
\mathbf{0} & \mathbf{A}
\end{array}\right] \underline{\mathbf{x}}_{k}+\underline{\mathbf{w}}_{k}, \quad \underline{\mathbf{w}}_{k} \sim \mathcal{N}\left(\underline{0},\left[\begin{array}{cc}
\mathbf{Q} & \mathbf{0} \\
\mathbf{0} & \mathbf{Q}
\end{array}\right]\right)
$$

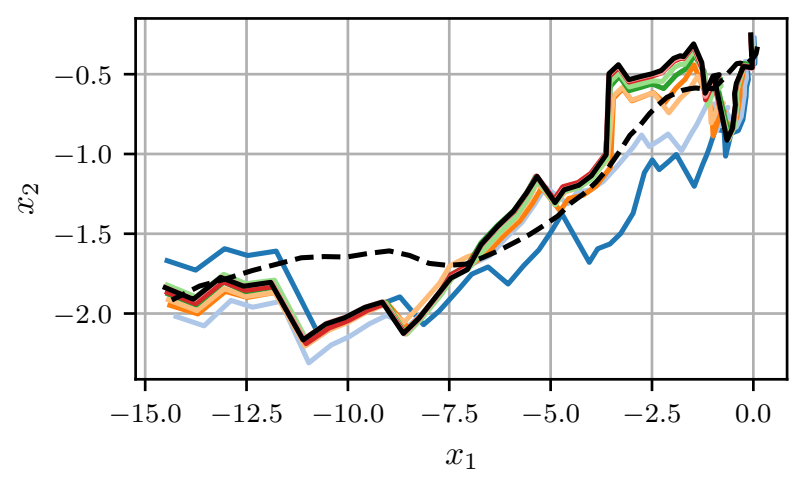

Fig. 5. For a single run, the trajectories estimated at sensor node $a$ are shown. The same quantizations (and color scheme) as in Fig. 4 are used. The dashed line is the true trajectory. affected by the zero-mean white Gaussian noise term $\underline{\mathbf{w}}_{k}$. The six-dimensional state $\underline{\mathbf{x}}_{k}$ consists of position, velocity, and acceleration in both the $x_{1}$ - and $x_{2}$-direction. The corresponding matrices of the process model are given by

$$
\mathbf{A}=\left[\begin{array}{ccc}
1 & \tau & \frac{1}{2} \tau^{2} \\
0 & 1 & \tau \\
0 & 0 & 1
\end{array}\right], \quad \mathbf{Q}=0.5\left[\begin{array}{ccc}
\frac{1}{20} \tau^{5} & \frac{1}{8} \tau^{4} & \frac{1}{6} \tau^{3} \\
\frac{1}{8} \tau^{4} & \frac{1}{3} \tau^{3} & \frac{1}{2} \tau^{2} \\
\frac{1}{6} \tau^{3} & \frac{1}{2} \tau^{2} & \tau
\end{array}\right]
$$

where $\tau$ is the time step [30]. For the Monte Carlo simulation with 10000 runs, the initial states $\underline{\mathbf{x}}_{0}$ are drawn from

$$
\underline{\mathbf{x}}_{0} \sim \mathcal{N}\left(\left[\begin{array}{c}
0 \\
0 \\
0.2 \\
0 \\
0 \\
0.3
\end{array}\right],\left[\begin{array}{cccccc}
0.5 & 0 & 0 & 0 & 0 & 0 \\
0 & 0.1 & 0 & 0 & 0 & 0 \\
0 & 0 & 0.05 & 0 & 0 & 0 \\
0 & 0 & 0 & 0.5 & 0 & 0 \\
0 & 0 & 0 & 0 & 0.1 & 0 \\
0 & 0 & 0 & 0 & 0 & 0.05
\end{array}\right]\right) \text {. }
$$

Two sensor nodes $a$ and $b$ are simulated that observe projections of position and velocity according to

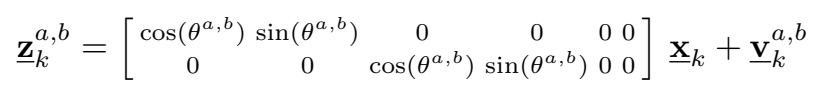

with $\theta^{a}=\frac{\pi}{4}, \theta^{b}=-\frac{\pi}{8}$. The zero-mean white Gaussian measurement noise terms $\underline{\mathbf{v}}_{k}^{a}, \underline{\mathbf{v}}_{k}^{b}$ have the covariance matrix

$$
\mathbf{R}^{a}=0.5\left[\begin{array}{cc}
1 & 0 \\
0 & 0.1
\end{array}\right], \quad \mathbf{R}^{b}=0.8\left[\begin{array}{cc}
1 & 0 \\
0 & 0.5
\end{array}\right],
$$

respectively. Each sensor node uses a Kalman filter to compute estimates for 50 time steps. Sensor node $a$ transmits its state and error covariance estimate to sensor node $b$ at every $5^{\text {th }}$ time step. Prior to transmission it quantizes the estimates with the proposed method and codebook parameter $x_{\max }=30.0$. Node $b$ fuses its own estimate with the received one by employing FCI. Every $11^{\text {th }}$ time step, sensor node $b$ quantizes and transmits its state and error covariance estimate to node $a$, which again fuses it with its own estimate using FCI. The receiving node in both cases reinitializes its own estimate with the fusion result.

Fig. 4 and Fig. 5 compare QCI using different quantization levels against FCI using 64-bit floating point numbers (denoted as FCI). Lower compression rates are close to the estimates obtained through FCI with 64-bit floats. However, even a 6-bit quantization still yields reasonable results. Quantization using less than 6 bits per codeword leads to too conservative bounds on the covariance matrices that cannot be encoded using the given codebook.

\section{CONCLUSION}

Data transmission capabilities in wireless sensor networks are limited by the amount of shared wireless bandwidth and the energy budget of individual sensor nodes. Algorithms for information fusion, such as fast covariance intersection, require the exchange of estimates and, in some cases, covariance matrices between wireless sensor nodes. If covariance matrices need to be transmitted, they dominate the amount of transmitted data. In this paper, we propose an efficient method for unbiased quantization of estimates and conservative quantization of covariance matrices and apply it to fast covariance intersection. The presented approach retains unbiasedness and conservativeness of covariance intersection while reducing the amount of data that must be transmitted. 
We empirically demonstrate the effectiveness of the proposed covariance quantization method and of fast covariance intersection applied to estimates and covariance matrices quantized with the aforementioned approach. Further improvements in performance could be achieved by using varying, possibly data-dependent quantization resolutions for subsets of the elements of the considered covariance matrices. The proposed quantization scheme can also be applied to other sensor fusion algorithms like information matrix fusion or inverse covariance intersection. For future work, theoretical results concerning the convergence behaviour of state and covariance estimates when using QCI in a distributed setting are of interest. Conservative quantization schemes for covariance matrices that do not enforce diagonal dominance of the quantization error matrix are also relevant, as they allow for decreased conservatism. Lastly, conservative vector quantization schemes for covariance matrices are worth consideration.

\section{REFERENCES}

[1] N. Kimura and S. Latifi, "A Survey on Data Compression in Wireless Sensor Networks," in International Conference on Information Technology: Coding and Computing (ITCC'05) - Volume II, vol. 2, Apr. 2005, pp. 8-13.

[2] T. Rault, A. Bouabdallah, and Y. Challal, "Energy Efficiency in Wireless Sensor Networks: A Top-Down Survey," Computer Networks, vol. 67, pp. 104-122, Jul. 2014.

[3] J. Yick, B. Mukherjee, and D. Ghosal, "Wireless Sensor Network Survey," Computer Networks, vol. 52, no. 12, pp. 2292-2330, Aug. 2008.

[4] G. Tolle et al., "A Macroscope in the Redwoods," in Proceedings of the 3rd International Conference on Embedded Networked Sensor Systems (SenSys '05), San Diego, California, USA, Nov. 2005, pp. 51-63.

[5] G. Werner-Allen et al., "Deploying a Wireless Sensor Network on an Active Volcano," IEEE Internet Computing, vol. 10, no. 2, pp. 18-25, Mar. 2006.

[6] M. S. Jamil et al., "Smart Environment Monitoring System by Employing Wireless Sensor Networks on Vehicles for Pollution Free Smart Cities," Procedia Engineering, vol. 107, pp. 480-484, Jan. 2015.

[7] F. Osterlind et al., "Integrating Building Automation Systems and Wireless Sensor Networks," in 2007 IEEE Conference on Emerging Technologies and Factory Automation (EFTA 2007), Patras, Greece, Sep. 2007, pp. 1376-1379.

[8] T. Torfs et al., "Low Power Wireless Sensor Network for Building Monitoring," IEEE Sensors Journal, vol. 13, no. 3, pp. 909-915, Mar. 2013.

[9] C.-Y. Lin, W.-C. Peng, and Y.-C. Tseng, "Efficient In-Network Moving Object Tracking in Wireless Sensor Networks," IEEE Transactions on Mobile Computing, vol. 5, no. 8, pp. 1044-1056, Aug. 2006.

[10] H.-W. Tsai, C.-P. Chu, and T.-S. Chen, "Mobile Object Tracking in Wireless Sensor Networks," Computer Communications, vol. 30, no. 8, pp. 1811-1825, Jun. 2007.

[11] S. J. Julier and J. K. Uhlmann, "A Non-divergent Estimation Algorithm in the Presence of Unknown Correlations," in Proceedings of the IEEE American Control Conference (ACC 1997), vol. 4, Albuquerque, New Mexico, USA, Jun. 1997, pp. 2369-2373.

[12] W. Niehsen, "Information Fusion based on Fast Covariance Intersection Filtering," in Proceedings of the 5th International Conference on Information Fusion (Fusion 2002), Annapolis, Maryland, USA, Jul. 2002.
[13] D. Fränken and A. Hüpper, "Improved Fast Covariance Intersection for Distributed Data Fusion," in Proceedings of the 8th International Conference on Information Fusion (Fusion 2005), Philadelphia, Pennsylvania, USA, Jul. 2005.

[14] B. Noack et al., "Decentralized Data Fusion with Inverse Covariance Intersection," Automatica, vol. 79, pp. 35-41, May 2017.

[15] B. Noack, J. Sijs, and U. D. Hanebeck, "Inverse covariance intersection: New insights and properties," in Proceedings of the 20th International Conference on Information Fusion (Fusion 2017), Xi' an, China, July 2017.

[16] J. Sijs, M. Lazar, and P. P. J. van den Bosch, "State-fusion with Unknown Correlation: Ellipsoidal Intersection," in Proceedings of the 2010 American Control Conference (ACC 2010), Baltimore, Maryland, USA, Jun. 2010.

[17] B. Noack, J. Sijs, and U. D. Hanebeck, "Algebraic analysis of data fusion with ellipsoidal intersection," in Proceedings of the 2016 IEEE International Conference on Multisensor Fusion and Integration for Intelligent Systems (MFI 2016), Baden-Baden, Germany, September 2016.

[18] T. Srisooksai et al., "Practical Data Compression in Wireless Sensor Networks: A Survey," Journal of Network and Computer Applications, vol. 35, no. 1, pp. 37-59, Jan. 2012.

[19] J.-J. Xiao and Z.-Q. Luo, "Decentralized Estimation in an Inhomogeneous Sensing Environment," IEEE Transactions on Information Theory, vol. 51, no. 10, pp. 3564-3575, Oct. 2005.

[20] J.-J. Xiao et al., "Power Scheduling of Universal Decentralized Estimation in Sensor Networks," IEEE Transactions on Signal Processing, vol. 54, no. 2, pp. 413-422, Feb. 2006.

[21] J. Li and G. AlRegib, "Rate-Constrained Distributed Estimation in Wireless Sensor Networks," IEEE Transactions on Signal Processing, vol. 55, no. 5, pp. 1634-1643, May 2007.

[22] — , "Distributed Estimation in Energy-Constrained Wireless Sensor Networks," IEEE Transactions on Signal Processing, vol. 57, no. 10, pp. 3746-3758, Oct. 2009.

[23] A. Ribeiro, G. B. Giannakis, and S. I. Roumeliotis, "SOI-KF: Distributed Kalman Filtering With Low-Cost Communications Using the Sign of Innovations," IEEE Transactions on Signal Processing, vol. 54, no. 12, pp. 4782-4795, Dec. 2006.

[24] E. J. Msechu et al., "Decentralized Quantized Kalman Filtering With Scalable Communication Cost," IEEE Transactions on Signal Processing, vol. 56, no. 8, pp. 3727-3741, Aug. 2008.

[25] R. Forsling et al., "Consistent Distributed Track Fusion Under Communication Constraints," in Proceedings of the 22nd International Conference on Information Fusion (Fusion 2019), Ottawa, Canada, Jul. 2019.

[26] M. Hopkins et al., "Stochastic Rounding and Reduced-Precision Fixed-Point Arithmetic for Solving Neural Ordinary Differential Equations," Philosophical Transactions of the Royal Society A: Mathematical, Physical and Engineering Sciences, vol. 378, no. 2166, Mar. 2020.

[27] S. Gupta et al., "Deep Learning with Limited Numerical Precision," arXiv:1502.02551v1 [cs.LG], Feb. 2015.

[28] B. Widrow, I. Kollar, and M.-C. Liu, "Statistical theory of quantization," IEEE Transactions on Instrumentation and Measurement, vol. 45, no. 2, pp. 353-361, Apr. 1996, conference Name: IEEE Transactions on Instrumentation and Measurement.

[29] R. A. Horn and C. R. Johnson, Matrix Analysis, 2nd ed. Cambridge University Press, 2012.

[30] X. R. Li and V. Jilkov, "Survey of maneuvering target tracking. Part I. Dynamic models," IEEE Transactions on Aerospace and Electronic Systems, vol. 39, no. 4, pp. 1333-1364, Oct. 2003, conference Name: IEEE Transactions on Aerospace and Electronic Systems. 